\title{
Numerical Simulation of 2D Transonic Flows in DCA 8\% Cascade
}

\author{
Jaroslav Huml ${ }^{1, a}$, Karel Kozel ${ }^{1}$, and Jaromír Př́íhoda ${ }^{2}$ \\ 1 Czech Technical University in Prague, Faculty of Mechanical Engineering \\ 2 Academy of Sciences of the Czech Republic, Institute of Thermomechanics
}

\begin{abstract}
This work deals with a numerical simulation of 2D inviscid and laminar compressible flows in the DCA $8 \%$ cascade achieved by the FVM using a multistage Runge-Kutta method and Lax-Wendroff scheme (Richtmyer form) with an artificial dissipation on non-orthogonal structured grids. The results are discussed and compared with other similar ones and experiment.
\end{abstract}

\section{Introduction}

The authors deal with development of their own software that could be applied in general for the simulation of any flow, i.e. an inviscid and a viscous (both laminar and turbulent) flow. It was decided the software would include FVM schemes - a multistage Runge-Kutta method and Lax-Wendroff scheme with a Jameson artificial dissipation. This software has been successfully tested on the simulation of inviscid and laminar flows in a test case, in the GAMM channel. Now the authors use it for numerical solutions to inviscid and laminar flows in a DCA $8 \%$ cascade.

\section{Mathematical models}

\subsection{Navier-Stokes equations}

A general two-dimensional flow of a compressible laminar fluid (the authors assumed that the flowing medium was a calorically perfect gas - the Newtonian fluid.) is described by the system of Navier-Stokes equations

$$
\mathbf{W}_{t}+\mathbf{F}_{x}+\mathbf{G}_{y}=\mathbf{R}_{x}+\mathbf{S}_{y}
$$

where $^{1}$

$$
\begin{aligned}
\mathbf{W} & =(\rho, \rho u, \rho v, e)^{T}, \\
\mathbf{F} & =\left(\rho u, \rho u^{2}+p, \rho u v,(e+p) u\right)^{T}, \\
\mathbf{G} & =\left(\rho v, \rho u v, \rho v^{2}+p,(e+p) v\right)^{T}, \\
\mathbf{R} & =\left(0, \tau_{x x}, \tau_{x y}, u \tau_{x x}+v \tau_{x y}-q_{x}\right)^{T}, \\
\mathbf{S} & =\left(0, \tau_{x y}, \tau_{y y}, u \tau_{x y}+v \tau_{y y}-q_{y}\right)^{T}
\end{aligned}
$$

\footnotetext{
${ }^{a}$ e-mail: jar.huml@gmail.com

$1 \rho$ denotes density of the fluid; $(u, v, w)$ are components of the local velocity respectively in the direction of axis $x, y, z ; p$ is pressure (given by an equation of state); $e$ denotes density of total energy per a unit volume; $\tau_{i j}$ represents shear stress (assuming Stokes Law for mono-atomic gases); $q_{j}$ is heat flux (given by Fourier's Law assuming Mayer's formula); $\mu$ represents dynamical viscosity; $\gamma$ denotes the isentropic exponent (equalled to 1.4 for mono-atomic gases - air); and $\mathrm{Pr}$ is laminar Prandtl number (equalled to 0.9).
}

and

$$
\begin{aligned}
p & =(\gamma-1)\left[e-\frac{1}{2}\left(u^{2}+v^{2}\right)\right], \\
\tau_{i j} & =\mu\left(\frac{\partial u_{i}}{\partial x_{j}}+\frac{\partial u_{j}}{\partial x_{i}}\right)-\frac{1}{3} \delta_{i j} \frac{\partial u_{k}}{\partial x_{k}}, \\
q_{j} & =-\frac{\gamma}{\gamma-1} \frac{\mu}{\operatorname{Pr}} \frac{\partial}{\partial x_{j}}\left(\frac{p}{\rho}\right) .
\end{aligned}
$$

\subsection{Euler equations}

Assuming an inviscid fluid ( $\mu=0$ in viscous fluxes $\mathbf{R}, \mathbf{S}$ ), the system of Euler equations is used instead of the system of Navier-Stokes equations

$$
\mathbf{W}_{t}+\mathbf{F}_{x}+\mathbf{G}_{y}=0
$$

\subsection{Transformation to the dimensionless form}

All variables were considered dimensionless and inflow variables (.) $)_{\infty}$ were used as the reference variable for the transformation of the equations to dimensionless form (e.g. $\left.\bar{\rho}=\rho / \rho_{\infty}\right)$. Thus after the transformation the system of Euler equations is written in the same form, but the transformed system of Navier-Stokes equations ${ }^{2}$ (5) is a little different from aforementioned (1)

$$
\mathbf{W}_{t}+\mathbf{F}_{x}+\mathbf{G}_{y}=\frac{M a_{\infty}}{R e_{\infty}}\left(\mathbf{R}_{x}+\mathbf{S}_{y}\right)
$$

\section{Numerical methods}

For simulation of the flows mentioned previously, two finite volume method numerical schemes were applied to the cell centered form on non-orthogonal structured grids of quadrilateral or hexahedral cells $D_{i j}{ }^{3}$.

\footnotetext{
$2 M a_{\infty}, R e_{\infty}$ is an inlet Mach and Reynolds number.

3 In both schemes $\left|D_{i j}\right|$ represents the surface or volume of the cell.
} 
- Lax-Wendroff scheme in Richtmyer's predictor-corrector form $(L W)$

predictor step

$$
\mathbf{W}_{i j}^{n+1 / 2}=\mathbf{W}_{i j}^{n}-\frac{\Delta t}{2\left|D_{i j}\right|} \sum_{k=1}^{4}\left(\tilde{\mathbf{F}}_{k}^{n} \Delta y_{k}-\tilde{\mathbf{G}}_{k}^{n} \Delta x_{k}\right),
$$

corrector step

$$
\begin{aligned}
& \mathbf{W}_{i j}^{n+1}=\mathbf{W}_{i j}^{n}-\frac{\Delta t}{\left|D_{i j}\right|} \sum_{k=1}^{4}\left(\tilde{\mathbf{F}}_{k}^{n+1 / 2} \Delta y_{k}-\tilde{\mathbf{G}}_{k}^{n+1 / 2} \Delta x_{k}\right) \\
& +A D\left(\mathbf{W}_{i j}^{n}\right)
\end{aligned}
$$

- multistage Runge-Kutta method $(R K)$

$$
\begin{aligned}
\operatorname{Res} \mathbf{W}_{i j}^{(r)}= & \frac{1}{\left|D_{i j}\right|} \sum_{k=1}^{4}\left(\tilde{\mathbf{F}}_{k}^{r} \Delta y_{k}-\tilde{\mathbf{G}}_{k}^{r} \Delta x_{k}\right), \\
\mathbf{W}_{i j}^{(0)}= & \mathbf{W}_{i j k}^{n}, \\
\mathbf{W}_{i j k}^{(r+1)}= & \mathbf{W}_{i j k}^{(0)}-\alpha_{r} \Delta t \operatorname{Res} \mathbf{W}_{i j k}^{(r)}+A D\left(\mathbf{W}_{i j k}^{n}\right), \\
\mathbf{W}_{i j k}^{n+1}= & \mathbf{W}_{i j k}^{(3)}, \\
& \alpha_{0,1}=0.5, \alpha_{2}=1, r=0,1,2 .
\end{aligned}
$$

Numerical approximations $\tilde{\mathbf{F}}, \tilde{\mathbf{G}}$ of convective terms $\mathbf{F}, \mathbf{G}$ were considered in the forward form of the first order of accuracy. Numerical approximations $\tilde{\mathbf{R}}, \tilde{\mathbf{S}}$ of dissipative terms $\mathbf{R}, \mathbf{S}$ were approximated in the central form of the second order of accuracy and by using dual cells and applying Green's formula.

Both schemes were used for modelling the two-dimensional flow in the GAMM channel. For other mentioned simulations only the multistage Runge-Kutta method was used.

\subsection{Artificial dissipation}

Each scheme was extended by including Jameson's artificial dissipation to improve the stability of the method.

$$
\begin{aligned}
A D\left(\mathbf{W}_{i, j}^{n}\right) & =C_{1} \psi_{1}\left(\mathbf{W}_{i-1, j}^{n}-2 \mathbf{W}_{i, j}^{n}+\mathbf{W}_{i+1, j}^{n}\right) \\
& +C_{2} \psi_{2}\left(\mathbf{W}_{i, j-1}^{n}-2 \mathbf{W}_{i, j}^{n}+\mathbf{W}_{i, j+1}^{n}\right),
\end{aligned}
$$

where

$$
\begin{aligned}
& \psi_{1}=\frac{\left|p_{i-1, j}^{n}-2 p_{i, j}^{n}+p_{i+1, j}^{n}\right|}{\left|p_{i-1, j}^{n}\right|+\left|p_{i, j}^{n}\right|+\left|p_{i+1, j}^{n}\right|}, \\
& \psi_{2}=\frac{\left|p_{i, j-1}^{n}-2 p_{i, j}^{n}+p_{i, j+1}^{n}\right|}{\left|p_{i, j-1}^{n}\right|+\left|p_{i, j}^{n}\right|+\left|p_{i, j+1}^{n}\right|} .
\end{aligned}
$$

\section{Formulation of the problems}

The authors took into account the numerical simulations of 2D inviscid and laminar compressible flows in a DCA $8 \%$ cascade. The outline of the computational domain is shown in Figure 1. The left and right outlines $(\overline{A H}, \overline{D E})$ represent an inlet and outlet of the domain respectively. The bottom and top outlines are divided into two straight lines $(\overline{A B}, \overline{C D}, \overline{E F}, \overline{G H})$ that are free walls - the parts of the boundary where were applied a periodicity condition and curves $(\widehat{B C}, \widehat{F G})$ that represent the bottom/top part of DCA $8 \%$ profile - here were prescribed boundary conditions for a solid wall according to the mentioned type of flow (an inviscid or a viscous flow).

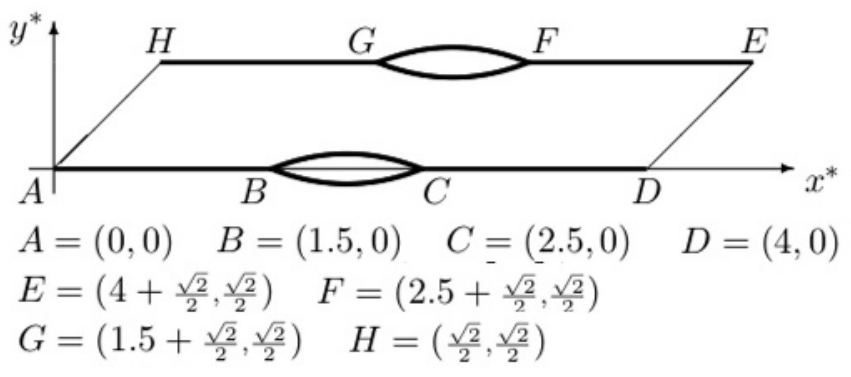

Fig. 1. Geometry of the DCA $8 \%$ cascade

This region was covered by two different meshes, nonorthogonal structured meshes with quadrangular cells (see Figure 2). The authors made such a convenient refinement of mesh near the bottom and top boundaries for a better detection of viscosity influence in the case of the laminar flows.

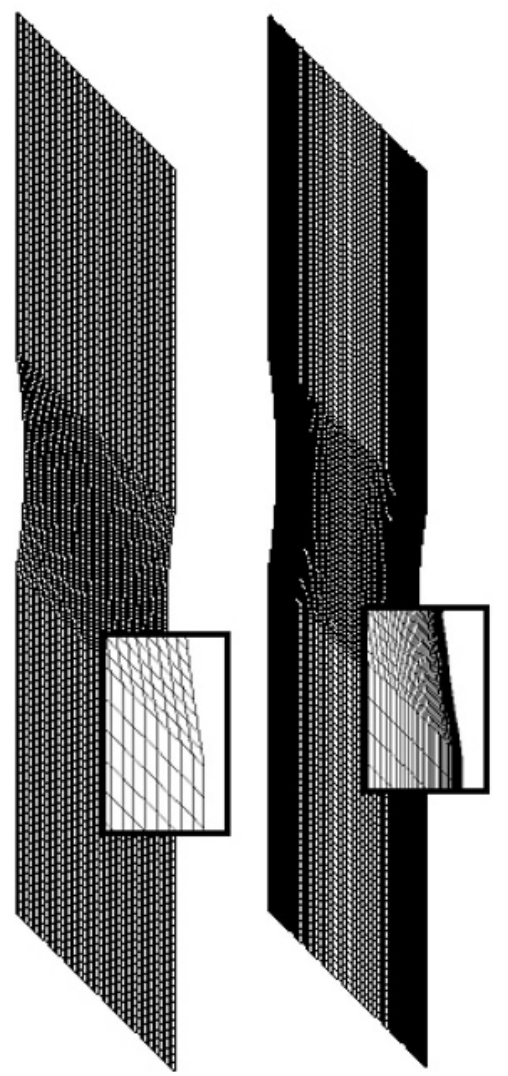

Fig. 2. Meshes used - 130 cells in the streamwise direction and 50 cells in the wall-normal direction (inviscid flows, left) and 170 cells in the streamwise direction and 120 cells in the wallnormal direction (laminar flows, right). The pictures were rotated by $90^{\circ}$ for a better resolution. 


\subsection{Boundary conditions}

- inlet: $\rho_{1}=1, u_{1}=M a_{1} \cos \alpha, v_{1}=M a_{1} \sin \alpha, p_{1}$ was extrapolated from the flow field and $e_{1}$ was calculated using the equation of state.

- outlet: $p_{2}$ was prescribed and the other components were extrapolated from the flow field or calculated.

- solid wall: velocity components were prescribed so that the sum of velocity vectors equals zero (viscous flows $u=v=0$ ) or equals zero in their tangential component (inviscid flows $-(u, v) \mathbf{n}=0$ ).

- periodicity: the value of the variable in the cell at the bottom/top part of the boundary corresponds to the value in the cell from the flow field near the top/bottom part of the boundary.

\section{Numerical results and discussion}

As mentioned above, the authors have dealt with the numerical simulation of 2D inviscid and laminar transonic flows in the DCA 8\% cascade and applied the multistage Runge-Kutta method, Lax-Wendroff scheme in Richtmyer's predictor-corrector form and two non-orthogonal structured grids with $130 \times 50$ and $170 \times 120$ cells respectively (see Figure 2) for its modelling. The authors considered a few different values of inlet Mach number $M a_{\infty} \in(0.8,1.2)$, Reynolds numbers $R e_{\infty} \in\left(10^{5}, 10^{6}\right)$ (in laminar flows) and angles of attack $\alpha \in\left(-3^{\circ}, 3^{\circ}\right)$.

The numerical results are presented in Mach number isolines. The results for inviscid flows are shown in Figures 3 (left) and 5 (left). The result for laminar flows is printed in Figure 6 (left).

For verification of the results achieved by a software developed by the authors, all the results were compared with P. Pořízková results ([4], see Figures 3 (right), 5 (right) and 6 (right)) and the experiment carried out by R. Dvořák ([1], see Figures 4 and 7) at the Institute of Thermodynamics of Academy of Sciences of the Czech Republic.

Based on the comparisons above with other similar numerical results or experiments, the software developed by the authors has proved itself (the good mutual agreement was found in all the simulated cases) and it will be used in a turbulence modelling.

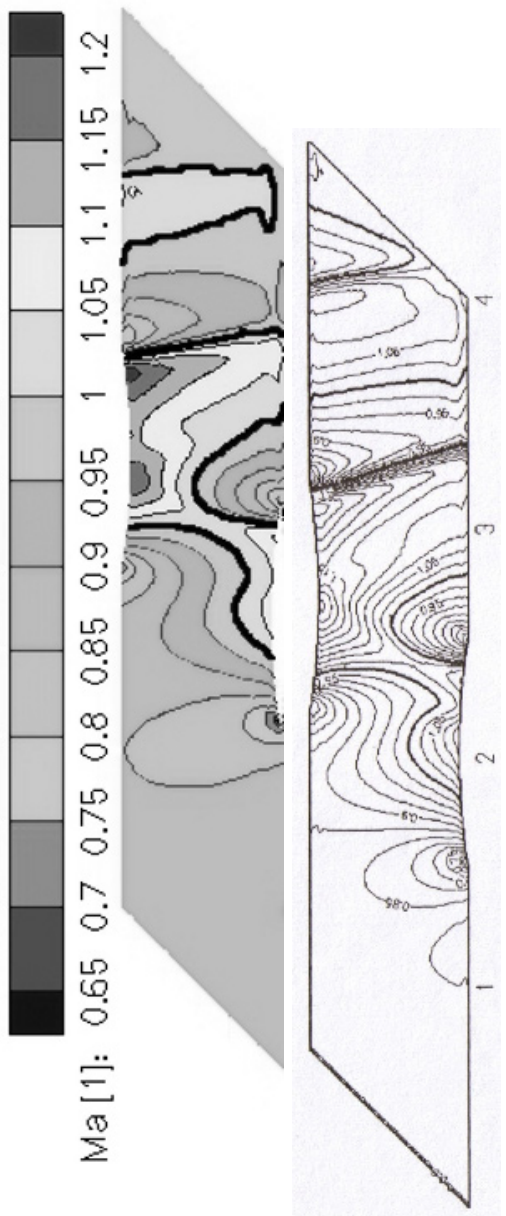

Fig. 3. Inviscid compressible flow in the DCA $8 \%$ cascade at $M a_{\infty}=0.92$ : Mach number isolines $-R K$ scheme, $\alpha=2^{\circ}$, mesh with $130 \times 50$ cells (left) and MacCormack scheme, $\alpha=1.2^{\circ}$, mesh with $150 \times 50$ cells, adopted from [4] (right). The pictures were rotated by $90^{\circ}$ for a better resolution.

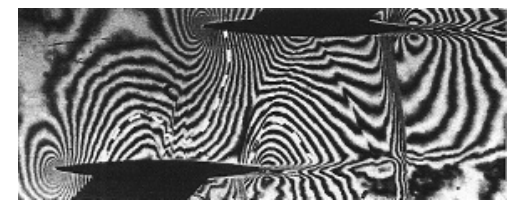

Fig. 4. Experiment in the DCA $8 \%$ cascade at $M a_{\infty}=$ $0.863, R e_{\infty}=10^{6}$ and $\alpha=0^{\circ}$ : Mach number isolines - adopted from [1].

\section{Conclusions}

This article presents a few results achieved by using the software with the implemented FVM Lax-Wendroff scheme and multistage Runge-Kutta method with Jameson's artificial dissipation for the simulation of a 2D transonic flow of the inviscid and laminar compressible fluid in the DCA $8 \%$ cascade. Numerical results show the good agreement with other numerical results (e.g. P. Pořízková [4]) and experimental results carried out at the Institute of Thermodynamics AS CZ. 


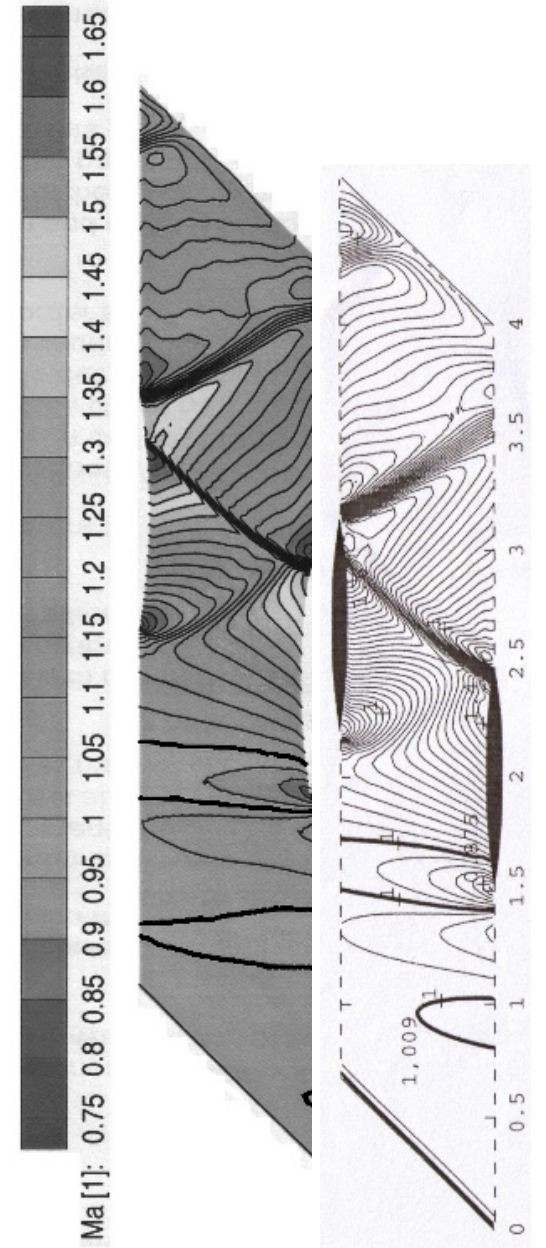

Fig. 5. Inviscid compressible flow in the DCA $8 \%$ cascade at $M a_{\infty}=1.12$ : Mach number isolines $-R K$ scheme, $\alpha=0.5^{\circ}$, mesh with $130 \times 50$ cells (left) and Composite scheme, $\alpha=0^{\circ}$, mesh with $170 \times 120$ cells, adopted from [4] (right). The pictures were rotated by $90^{\circ}$ for a better resolution.

\section{Acknowledgments}

This work was partly supported by grants No. P101/10/1329 and P101/12/1271 of the Grant Agency of the Czech Republic, grant No. IAA200760801 of the Grant Agency of the Academy of Science of the Czech Republic and grant No. SGS 10/243/OHK2/3T/12 of the Student Grant Competition of Czech Technical University in Prague.

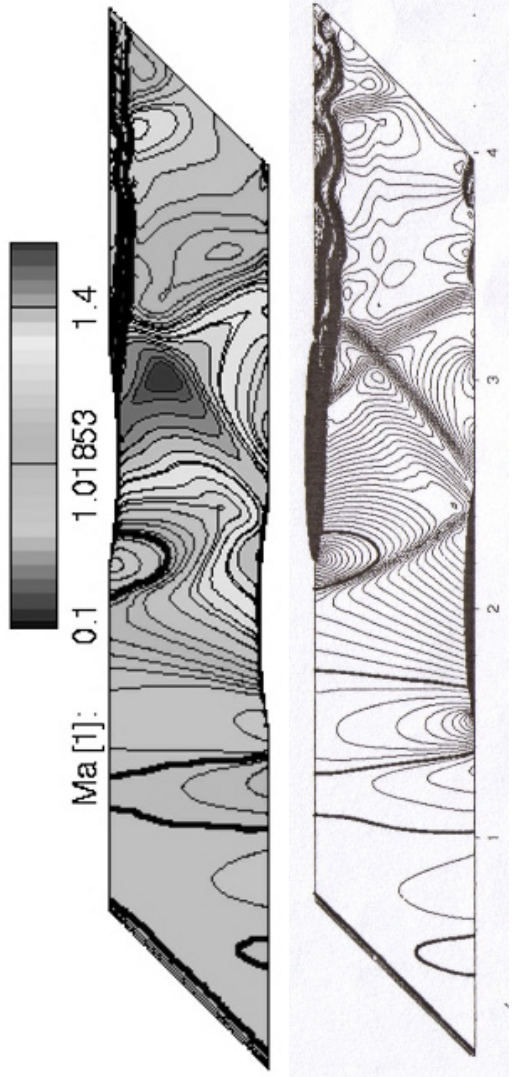

Fig. 6. Laminar compressible flow in the DCA $8 \%$ cascade at $M a_{\infty}=1.1$ and $R e_{\infty}=2.1 \cdot 10^{6}$ : Mach number isolines $-R K$ scheme, $\alpha=0^{\circ}$, mesh with $170 \times 120$ cells (left) and MacCormack scheme, $\alpha=0^{\circ}$, mesh with $320 \times 80$ cells, adopted from [4] (right). The pictures were rotated by $90^{\circ}$ for a better resolution.

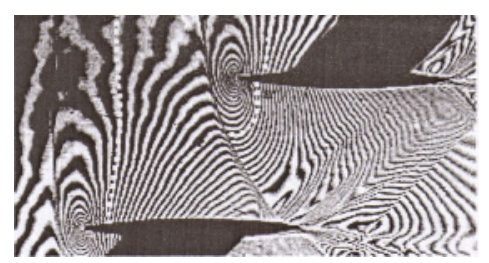

Fig. 7. Experiment in the DCA $8 \%$ cascade at $M a_{\infty}=$ $1.013, R e_{\infty}=10^{6}$ and $\alpha=0^{\circ}$ : Mach number isolines - adopted from [1].

\section{References}

1. R. Dvořák, Transonic Flows (Academia, Prague, 1986) [in Czech]

2. R. Dvořák, K. Kozel, Mathematical modelling in aerodynamics (CTU in Prague, Prague, 1996) [in Czech]

3. J. Huml, K. Kozel, J. Příhoda, Proc. 11th conference on Power System Engineering, Thermodynamics $\mathcal{E}$ Fluid Flow 2012 73-76 (University of West Bohemia, Pilsen, 2012)

4. P. Pořízková, Numerical Solution of Compressible Flows Using Finite Volume Method, (CTU in Prague, Prague, 2009) [Ph.D. Dissertation, in Czech] 